\title{
SUBGROUPS OF THE SCHUR MULTIPLIER
}

\author{
R. J. HIGGS
}

(Received 17 March 1989)

Communicated by $\mathrm{H}$. Lausch

\begin{abstract}
Two subgroups $M_{E}(G)$ and $M_{l}(G)$ of the Schur multiplier $M(G)$ of a finite group $G$ are introduced: $M_{E}(G)$ contains those cohomology classes $[\alpha]$ of $M(G)$ for which every element of $G$ is $\alpha$-regular, and $M_{I}(G)$ consists of those cohomology classes of $M(G)$ which contain a $G$-invariant cocycle. It is then shown that under suitable circumstances, such as when $G$ has odd order, that each element of $M_{I}(G)$ can be expressed as the product of an element of $M_{E}(G)$ and an element of the image of the inflation homomorphism from $M\left(G / G^{\prime}\right)$ into $M(G)$.
\end{abstract}

1980 Mathematics subject classification (Amer. Math. Soc.) (1985 Revision): 20 C 25.

Keywords and phrases: Schur multiplier.

\section{Introduction}

Throughout this paper $G$ will denote a finite group, $Z^{2}\left(G, \mathbf{C}^{*}\right)$ the group of normalized cocycles of $G$, and $M(G)$ the Schur multiplier of $G$. The reader unfamiliar with the Schur multiplier is referred to [5] for basic definitions and elementary results.

We begin by defining two subgroups of $M(G)$.

1. Let $\alpha \in Z^{2}\left(G, C^{*}\right)$, then an element $x$ of $G$ is said to be $\alpha$-regular if $\alpha(x, g)=\alpha(g, x)$ for all $g \in C_{G}(x)$.

We define $Z_{E}^{2}\left(G, \mathbf{C}^{*}\right)$ to be the subgroup of $Z^{2}\left(G, \mathbf{C}^{*}\right)$ containing those cocycles for which every element of $G$ is $\alpha$-regular. Now since an element of $G$ is $\alpha$-regular if and only if it is $\beta$-regular for any cocycle $\beta$ cohomologous

(C) 1990 Australian Mathematical Society $0263-6115 / 90 \$ A 2.00+0.00$ 
to $\alpha$, we may define a subgroup of $M(G)$ by

$$
M_{E}(G)=\left\{[\alpha] \in M(G): \alpha \in Z_{E}^{2}\left(G, C^{*}\right)\right\} .
$$

2. $G$ acts on $Z^{2}\left(G, C^{*}\right)$ via the action $\alpha \mapsto \alpha^{g}$, where $\alpha^{g}$ is the element of $Z^{2}\left(G, C^{*}\right)$ defined by $\alpha^{g}(x, y)=\alpha\left(g x g^{-1}, g y g^{-1}\right)$ for all $x, y \in G$. We define $Z_{I}^{2}\left(G, C^{*}\right)$ to be the group of $G$-invariant elements of $Z^{2}\left(G, \mathbf{C}^{*}\right)$, and $M_{I}(G)$ to be the subgroup of $M(G)$ consisting of those cohomology classes which contain an element of $Z_{I}^{2}\left(G, C^{*}\right)$.

The purpose of this note is to show how to calculate both $M_{E}(G)$ and $M_{I}(G)$, and to study the relationship between these two groups. In a previous paper [1], the author gave an example of a $p$-group for which $M_{E}(G)$ is non-trivial, thus negating an argument previously given by Mangold in [7]. The subgroup $M_{I}(G)$ has not been examined previously, probably because its very definition relies on making a 'clever' choice of cocycle within a cohomology class. Despite this we shall essentially show how to find $M_{I}(G)$ given $M\left(G / G^{\prime}\right)$ and $M_{E}(G)$, and then proceed to find these groups for some specific examples.

\section{Inflation of elements of $M_{I}(G)$}

Let $\alpha \in Z^{2}\left(G, \mathrm{C}^{*}\right)$. Then we define a function $f_{\alpha}: G \times G \rightarrow \mathrm{C}^{*}$ by $f_{\alpha}(g, x)=\alpha\left(g x, g^{-1}\right) \alpha(g, x) / \alpha\left(g, g^{-1}\right)$. It is easy to check that for all $\alpha$, $\beta \in Z^{2}\left(G, \mathbf{C}^{*}\right)$ and all $g, h, x, y \in G$ that

$$
\begin{aligned}
f_{\alpha} f_{\beta} & =f_{\alpha \beta} \\
f_{\alpha}(g, x) f_{\alpha}(g, y) \alpha^{g}(x, y) & =f_{\alpha}(g, x y) \alpha(x, y) ; \\
f_{\alpha}(g, x) f_{\alpha}\left(h, g x g^{-1}\right) & =f_{\alpha}(g h, x) .
\end{aligned}
$$

Our first result describes the elements of $Z_{E}^{2}\left(G, C^{*}\right)$ and $Z_{I}^{2}\left(G, C^{*}\right)$ in terms of $f_{\alpha}$.

LEMMA 1.1. (i) $\alpha \in Z_{I}^{2}\left(G, \mathbf{C}^{*}\right)$ if and only if $f_{\alpha} \in P\left(G, G, \mathbf{C}^{*}\right)$, the group of pairings of $G$ in $\mathbf{C}^{*}$.

(ii) $\alpha \in Z_{E}^{2}\left(G, \mathbf{C}^{*}\right)$ if and only if there exists a function $\delta: G \rightarrow \mathbf{C}^{*}$ such that $f_{\alpha}(g, x)=\delta(x) / \delta^{g}(x)$ for all $g, x \in G$, where $\delta^{g}(x)=\delta\left(g x g^{-1}\right)$.

(iii) $M_{E}(G)$ is a subgroup of $M_{I}(G)$.

Proof. From (2) we have that $\alpha \in Z_{I}^{2}\left(G, \mathbf{C}^{*}\right)$ if and only if $f_{\alpha}(g, \ldots)$ is a linear character of $G$ for each $g \in G$. If the latter condition is true we obtain in (3) that $f_{\alpha}\left(h, g x g^{-1}\right)=f_{\alpha}(h, x)$, so that in fact $f_{\alpha} \in P\left(G, G, C^{*}\right)$. 
Now let $\alpha, \beta \in Z^{2}\left(G, C^{*}\right)$ with $[\alpha]=[\beta]$. Then there exists a function $\delta: G \rightarrow C^{*}$ such that $\delta(x y) \beta(x, y)=\delta(x) \delta(y) \alpha(x, y)$ for all $x, y \in G$, and so $\delta^{g}(x) f_{\beta}(g, x)=\delta(x) f_{\alpha}(g, x)$ for all $g, x \in G$. However if $\alpha$ is a class-function cocycle then from (7.2.2) of [4], $\alpha \in Z_{E}^{2}\left(G, \mathbf{C}^{*}\right)$ if and only if $f_{\alpha}(g, x)=1$ for all $g, x \in G$. The fact that $M_{E}(G)$ is a subgroup of $M_{I}(G)$ now follows from the above observations.

As an immediate consequence of this result we obtain a necessary condition for $[\alpha] \in M_{I}(G)$ which is independent of the choice of cocycle from $[\alpha]$.

Corollary 1.2. Let $\alpha \in Z_{I}^{2}\left(G, \mathbf{C}^{*}\right)$. Then every element of $G^{\prime}$ is $\alpha$ regular.

Proof. By 1.1 we have that $f_{\alpha}(g, x)=1$ for all $x \in G^{\prime}$ and all $g \in G$. Thus if $x \in G^{\prime}$ and $g \in C_{G}(x)$ we obtain that $\alpha(g, x) / \alpha(x, g)=1$.

Obviously from 1.2 if $G$ is a perfect group then $M_{I}(G)=M_{E}(G)$. In the particular case when $G$ is the alternating group $A_{n}$, it is straightforward to verify from (2.12.5) of [5] that every element in the centre of the representation group of $A_{n}$ is a commutator, and hence we obtain that $M_{E}\left(A_{n}\right)=\{[1]\}$ by applying the next result, which shows how to compute $M_{E}(G)$ for any group $G$. We also note that for the symmetric group $S_{n}$, $M_{I}\left(S_{n}\right)=M_{E}\left(S_{n}\right)=\{[1]\}$, since the restriction homomorphism from $M\left(S_{n}\right)$ into $M\left(A_{n}\right)$ is injective.

Proposition 1.3. Let $(H, \pi)$ be a finite central extension of $G$ with $\operatorname{ker} \pi=A$. Let tra: $\operatorname{Irr}(A) \rightarrow M(G)$ be the transgression homomorphism, and $\operatorname{Com}(H)$ denote the set of commutators of elements of $H$. Then $\operatorname{tra}(\lambda) \in$ $M_{E}(G)$ if and only if $\operatorname{Com}(H) \cap A \subseteq \operatorname{ker} \lambda$.

Proof. Let $\{r(g): g \in G\}$ with $r(1)=1$ be a transversal of $A$ in $H$, so that for all $x, y \in G, r(x) r(y)=A(x, y) r(x y)$ for some element $A(x, y) \in$ $A$. Then $\operatorname{Com}(H) \cap A=\left\{A(x, y)(A(y, x))^{-1}: x, y \in G\right.$ with $\left.[x, y]=1\right\}$.

Now $\operatorname{tra}(\lambda)=[\alpha]$, where $\alpha(x, y)=\lambda(A(x, y))$ for all $x, y \in G$. Hence every element of $G$ is $\alpha$-regular if and only if $\operatorname{Com}(H) \cap A \subseteq \operatorname{ker} \lambda$.

Our main result which describes how to find $M_{l}(G)$ is based upon the following simple observation.

Corollary 1.4. Let $G$ be an abelian group. Then $M_{I}(G)=M(G)$ and $M_{E}(G)=\{[1]\}$.

Proof. Clearly any cocycle of $G$ is $G$-invariant so that $M_{I}(G)=M(G)$. 
Now let $[\alpha] \in M(G)$. Then there exists a finite central extension $(H, \pi)$ of $G$ with $\operatorname{ker} \pi=H^{\prime}$ and a faithful linear character $\lambda$ of $H^{\prime}$ such that $\operatorname{tra}(\lambda)=[\alpha]$. So by $1.3, \operatorname{tra}(\lambda) \in M_{E}(G)$ if and only if $H \cong G$, that is if and only if $[\alpha]=[1]$.

We have already shown in 1.2 that if $\alpha \in Z_{I}^{2}\left(G, \mathbf{C}^{*}\right)$ then every element of $G^{\prime}$ is $\alpha$-regular, so we might conjecture that each such $\alpha$ is inflated from an element of $Z^{2}\left(G / G^{\prime}, C^{*}\right)$. This unfortunately is false in general as shown in [1]. However our main result shows that $[\alpha]$ can be 'inflated' from $M\left(G / G^{\prime}\right)$ if we allow a more liberal interpretation of the term inflation.

THEOREM 1.5. Let inf: $M\left(G / G^{\prime}\right) \rightarrow M_{I}(G)$ be the inflation homomorphism, $S$ denote the image of the Sylow 2-subgroup $\bar{S}$ of $M\left(G / G^{\prime}\right)$ under inf, and let $n$ be the exponent of $S M_{E}(G) / M_{E}(G)$. Then for each $[\alpha] \in$ $M_{I}(G)$ there exists $[\beta] \in M\left(G / G^{\prime}\right)$ and $[\gamma] \in M_{I}(G)$ with $[\gamma]^{2 n} \in M_{E}(G)$ such that $[\alpha]=\inf ([\beta])[\gamma]$.

Proof. Define $F: M_{I}(G) \rightarrow M_{I}(G) / M_{E}(G)$ by $F([\alpha])=\left[f_{\alpha}\right] M_{E}(G)$ for $\alpha \in Z_{I}^{2}\left(G, C^{*}\right)$, so that $F$ is a well-defined homomorphism by 1.1 and (1). Also

$$
\begin{aligned}
\operatorname{ker} F= & \left\{[\alpha] \in M_{I}(G): \alpha(g, x) / \alpha(x, g)=\alpha(x, g) / \alpha(g, x)\right. \\
& \quad \text { for all }(g, x) \in G \times G \text { with }[g, x]=1\} \\
=\left\{[\alpha] \in M_{I}(G):[\alpha]^{2} \in M_{E}(G)\right\} . &
\end{aligned}
$$

Now the function $F$ induces homomorphisms $\bar{f}: M\left(G / G^{\prime}\right) \rightarrow M\left(G / G^{\prime}\right) / \bar{S}$ and $f: M_{I}(G) \rightarrow M_{I}(G) / S M_{E}(G)$ defined by $\bar{f}([\beta])=\left[f_{\beta}\right] \bar{S}$ and $f([\alpha])=$ $\left[f_{\alpha}\right] S M_{E}(G)$ for $\alpha \in Z_{I}^{2}\left(G, \mathbf{C}^{*}\right)$. In the former case $M_{E}\left(G / G^{\prime}\right)=\{[1]\}$ from 1.4, so that $\operatorname{ker} \bar{f}=\bar{S}$, from which it follows that $\bar{f}$ is surjective. In the latter case we have that if $\alpha \in Z_{I}^{2}\left(G, \mathbf{C}^{*}\right)$, then $\gamma\left(g G^{\prime}, x G^{\prime}\right)=f_{\alpha}(g, x)$ for all $g, x \in G$ is a well-defined cocycle of $G / G^{\prime}$ from 1.1. Thus $\operatorname{ker} f=$ $\left\{[\alpha] \in M_{I}(G):[\alpha]^{2 n} \in M_{E}(G)\right\}$, since $\left[f_{\alpha}\right]^{n}=\left[f_{\alpha^{n}}\right]$ for $\alpha \in Z_{I}^{2}\left(G, \mathbf{C}^{*}\right)$.

Finally inf induces a homomorphism $I: M\left(G / G^{\prime}\right) / \bar{S} \rightarrow M_{I}(G) / S M_{E}(G)$ defined by $I([\beta] \bar{S})=\inf ([\beta]) S M_{E}(G)$. This mapping renders the following diagram commutative

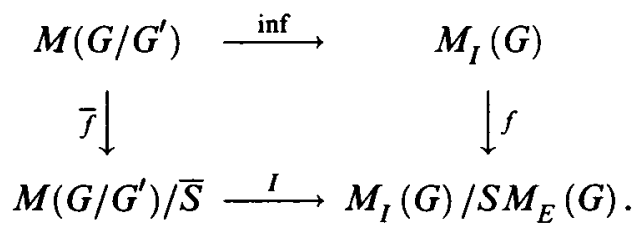


Now let $\alpha \in Z_{I}^{2}\left(G, \mathbf{C}^{*}\right)$, then as shown above $\left[f_{\alpha}\right] S M_{E}(G)=I([\gamma] \bar{S})$ for some $[\gamma] \in M\left(G / G^{\prime}\right)$. Also since $\bar{f}$ is surjective we have that $[\gamma] \bar{S}=\left[f_{\beta}\right] \bar{S}$ for some $[\beta] \in M\left(G / G^{\prime}\right)$. Thus we obtain that $f(\inf ([\beta]))=f([\alpha])$, and hence $\inf ([\beta])[\alpha]^{-1} \in \operatorname{ker} f$.

There are a number of special cases of 1.5 which justify a separate statement. The first is to just note that $\bar{S}$ is trivial if and only if $G / G^{\prime}$ has a cyclic Sylow 2-subgroup, and the second concerns an isomorphism implicitly defined in 1.5. For an abelian group $B$, let $B_{m}=\left\{b \in B: b^{m}=1\right\}$.

COROLlary 1.6. Let $T$ denote the image of $M\left(G / G^{\prime}\right)$ under the inflation homomorphism, $S$ be the Sylow 2-subgroup of $T$, and $n$ be the exponent of $S M_{E}(G) / M_{E}(G)$. Then

$$
\left(M_{I}(G) / M_{E}(G)\right) /\left(M_{I}(G) / M_{E}(G)\right)_{2 n} \cong T M_{E}(G) / S M_{E}(G) .
$$

Proof. We may easily modify the commutative diagram in 1.5 to obtain the following new commutative diagram

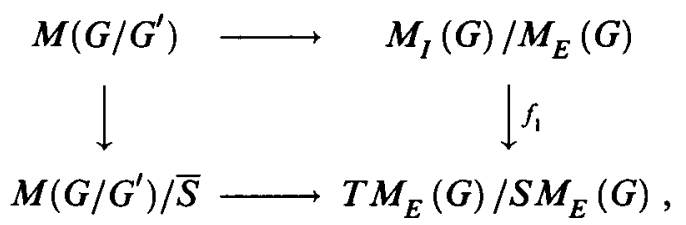

where $f_{1}\left([\alpha] M_{E}(G)\right)=\left[f_{\alpha}\right] S M_{E}(G)$ for $\alpha \in Z_{1}^{2}\left(G, \mathrm{C}^{*}\right)$, and the other homomorphisms are defined similarly in terms of those in 1.5. Now the homomorphisms on the left and bottom of the diagram are defined so as to be surjective, and hence $f_{1}$ is also surjective by commutativity. Finally $\operatorname{ker} f_{1}=\left(M_{I}(G) / M_{E}(G)\right)_{2 n}$.

We note in the context of 1.6 that $T \cong M\left(G / G^{\prime}\right) / H_{I}^{1}\left(G^{\prime}, \mathrm{C}^{*}\right)$ from the Lyndon-Hochschild-Serre exact sequence of cohomology, where $H_{I}^{1}\left(G^{\prime}, \mathbf{C}^{*}\right)$ denotes the $G$-invariant elements of $H^{1}\left(G^{\prime}, \mathrm{C}^{*}\right)$. We also observe that if $G / G^{\prime}$ is cyclic, then 1.6 yields that $M_{I}(G) / M_{E}(G)$ is an elementary abelian 2-group (possibly trivial).

The most interesting consequences of 1.5 occur when $G$ is a group of odd order, this situation is covered by the next result which summarizes the information obtained so far.

Corollary 1.7. Suppose that 2 does not divide the index of $M_{E}(G)$ in $M_{I}(G)$. Then for each $[\alpha] \in M_{I}(G)$ there exists $[\beta] \in M\left(G / G^{\prime}\right)$ and $[\gamma] \in$ $M_{E}(G)$ such that $[\alpha]=\inf ([\beta])[\gamma]$. In particular if $M_{E}(G)=\{[1]\}$, then $M\left(G / G^{\prime}\right) / H_{I}^{1}\left(G^{\prime}, \mathbf{C}^{*}\right) \cong M_{I}(G)$. 
There is one further result concerning the inflation of elements of $M_{I}(G)$ and $M_{E}(G)$ which was observed by the author in [1], and which we include here for the sake of completeness. Let $\operatorname{Proj}(G, \alpha)$ denote the set of irreducible projective characters of $G$ with cocycle $\alpha$.

Proposition 1.8. Let $\alpha \in Z^{2}\left(G, \mathbf{C}^{*}\right)$, and $N$ be a normal subgroup of $G$. Then $[\alpha]=\inf ([\beta])$ for some $[\beta] \in M(G / N)$ if and only if there exists a $G$-invariant element of degree 1 in $\operatorname{Proj}\left(N, \alpha_{N}\right)$.

\section{Examples}

In this section we shall compute $M_{E}(G)$, and where possible $M_{I}(G)$, for some specific types of group $G$. We start by considering semidirect products. Let $G$ be the semidirect product of a normal subgroup $N$ and a subgroup $T$. Now let $M_{E}(N)^{T}$ denote the $T$-invariant subgroup of $M_{E}(N)$, $\widetilde{M}_{E}(G)$ denote the kernel of the restriction homomorphism from $M_{E}(G)$ into $M_{E}(T)$, and $\widehat{N}=H^{1}\left(N, \mathrm{C}^{*}\right)$. Then using this notation we obtain the following result.

Proposition 2.1. Let $G$ be as above. Then

(i) $M_{E}(G) \cong M_{E}(T) \times \widetilde{M}_{E}(G)$;

(ii) there is an exact sequence

$$
\{[1]\} \rightarrow H \rightarrow \widetilde{M}_{E}(G) \stackrel{\text { res }}{\rightarrow} M_{E}(N)^{T} \rightarrow H^{2}(T, \widehat{N}),
$$

where $H \leq H^{1}(T, \hat{N})$, and res: $\widetilde{M}_{E}(G) \rightarrow M_{E}(N)^{T}$ is induced by the restriction homomorphism from $M_{E}(G)$ into $M_{E}(N)$.

Proof. The proof of this result is practically identical to that given in (2.2.5) of [5].

Since 2.1 is the direct analogue of the corresponding result for $M(G)$, we obtain the 'standard' corollaries to it. For example if $N$ is a normal Hall-subgroup of $G$, and $T$ is a complement of $N$ in $G$. Then $M_{E}(G) \cong$ $M_{E}(T) \times M_{E}(N)^{T}$.

The situation however becomes considerably more interesting when we consider direct products.

COROLlary 2.2. Let $N$ and $T$ be finite groups. Then

$$
M_{E}(N \times T) \cong M_{E}(N) \times M_{E}(T)
$$


and

$$
M_{I}(N \times T) \cong M_{I}(N) \times M_{I}(T) \times(N \otimes T)
$$

Proof. From the proof of (2.2.5) of [5] we have that the group $H$ of 2.1(ii) may be identified with those elements $[\alpha]$ of $\widetilde{M}_{E}(G)$ which contain a normal cocycle $\alpha$ with $\alpha(T, T)=\alpha(N, N)=1$. For such a cocycle we must have $\alpha\left(t, n^{\prime}\right)=\alpha\left(t^{\prime}, n\right)$ for all $n, n^{\prime} \in N$, and all $t, t^{\prime} \in T$ with $\left[n t, n^{\prime} t^{\prime}\right]=1$.

Here of course we have that $[N, T]=\{1\}$. So setting $n=1$, and $t^{\prime}=t$ we obtain that $\alpha\left(t, n^{\prime}\right)=1$ for all $t \in T$, and all $n^{\prime} \in N$; and hence $\alpha=1$. Thus $H$ is trivial, and so it follows from 2.1 that $M_{E}(N \times T) \cong$ $M_{E}(N) \times M_{E}(T)$.

An alternative proof is to just suitably adapt the proof of (2.3.13) of [4], this technique also yields that $M_{I}(N \times T) \cong M_{I}(N) \times M_{I}(T) \times(N \otimes T)$.

We now focus our attention on metacyclic groups.

Proposition 2.3. Let $G$ be a metacyclic group. Then $M_{E}(G)=\{[1]\}$ and $M_{I}(G) \cong M\left(G / G^{\prime}\right) / H_{I}^{1}\left(G^{\prime}, \mathbf{C}^{*}\right)$.

Proof. Let $G=\left\langle a, b: a^{m}=1, b^{s}=a^{t}, b a b^{-1}=a^{r}\right\rangle$, where the positive integers $m, r, s$ and $t$ satisfy $r^{s} \equiv 1(\bmod m), t \mid m$, and $m \mid t(r-1)$. Let $\left[\alpha^{\prime}\right] \in M(G)$, then from the proof of (2.11.3) of [5], there exists $\alpha \in\left[\alpha^{\prime}\right]$ with $\alpha\left(a^{i} b^{j}, a^{k} b^{l}\right)=\varepsilon^{k\left(r^{j}-1\right) /(r-1)}$, where $\varepsilon$ is an $n$th root of unity and $m /(r-1, m)$ divides $n$, which in turn divides $\left(1+\cdots+r^{s-1}, t\right)$. Moreover $[\alpha]=[1]$ if and only if $n=m /(r-1, m)$.

Now $a^{c} \in Z(G)$, where $c=m /(r-1, m)$; and hence $a^{c}$ is $\alpha$-regular if and only if $c\left(r^{l}-1\right) /(r-1) \equiv 0(\bmod n)$ for all $l$. However taking $l=1$, we obtain that $n=m /(r-1, m)$. Thus $M_{E}(G)=\{[1]\}$.

Finally suppose $[\alpha] \in M_{I}(G)$. Then from 1.2 every element of $G^{\prime}$ is $\alpha$-regular, and hence from $(1.10)$ of $[1]$ and $1.8,[\alpha]=\inf ([\beta])$ for some $[\beta] \in M\left(G / G^{\prime}\right)$; from which the desired result follows.

For our final example we shall look at extra special $p$-groups and dihedral groups, but consider where convenient only those cases for which $M(G)$ is non-trivial.

Proposition 2.4. (i) Let $G$ be an extra special p-group of order $p^{2 n+1}$. Then $M_{E}(G)=\{[1]\}$, and

$$
M_{I}(G)= \begin{cases}M(G), & \text { if } n>1 \\ \{[1]\}, & \text { if } n=1 .\end{cases}
$$


(ii) Let $D$ be the dihedral group of order $4 n$. Then $M_{E}(D)=\{[1]\}$, and

$$
M_{I}(D)= \begin{cases}M(D), & \text { if } n \text { is odd } \\ \{[1]\}, & \text { if } n \text { is even } .\end{cases}
$$

Proof. (i) Suppose $\alpha \in Z_{E}^{2}\left(G, \mathbf{C}^{*}\right)$, with $[\alpha] \neq[1]$. Then $G$ has $p^{2 n}+$ $p-1$ conjugacy classes from (V.16.14) of [2]. Thus since each element of $\operatorname{Proj}(G, \alpha)$ has degree at least $p$ we must have that $\left(p^{2 n}+p-1\right) p^{2} \leq p^{2 n+1}$, which is clearly impossible. Hence $M_{E}(G)=\{[1]\}$.

Now since $Z(G)=G^{\prime} \cong C_{p}$, we have from 1.8 that $[\alpha] \in M_{I}(G)$ if and only if $[\alpha]=\inf ([\beta])$ for some $[\beta] \in M\left(G / G^{\prime}\right)$. Here however the image of inflation is isomorphic to

$$
M\left(G / G^{\prime}\right) / C_{p} \cong \begin{cases}M(G), & \text { if } n>1 \\ \{[1]\}, & \text { if } n=1 .\end{cases}
$$

(ii) $M_{E}(D)=\{[1]\}$ is immediate from 2.3. Also from (1.10) of [1] and $1.8,[\alpha] \in M_{I}(D)$ if and only if every element of $D^{\prime}$ is $\alpha$-regular. However from (3.7.3) of [4] every element of $D^{\prime}$ is $\alpha$-regular if $n$ is odd for all $[\alpha] \in M(D)$, whereas if $n$ is even the element $a^{n}$ is $\alpha$-regular if and only if $[\alpha]=[1]$.

The reader may be somewhat disappointed at this stage that all the examples we have given have $M_{E}(G)=\{[1]\}$, however Macdonald in [6] has shown how to construct examples of $p$-groups where this is certainly not the case. Neither have we exploited the fact that if $\alpha \in Z_{E}^{2}\left(G, \mathbf{C}^{*}\right)$, then $\operatorname{Proj}(G, \alpha)$ behave similarly to $\operatorname{Irr}(G)$. We give here just one example of this similarity to give a flavour of this type of result. Its proof is essentially the same as that given for (6.34) of [3], provided we use (1.8) of [1].

Lemma 2.5. Let $G$ be a Frobenius group with Frobenius kernel $N$. Suppose that every element of $N$ is $\alpha$-regular for some cocycle $\alpha$ of $G$, and that there exists $\zeta \in \operatorname{Proj}\left(N, \alpha_{N}\right)$ which is $G$-invariant. Then

(i) if $\varphi \in \operatorname{Proj}\left(N, \alpha_{N}\right)$ with $\varphi \neq \zeta$, then $\varphi^{G} \in \operatorname{Proj}(G, \alpha)$;

(ii) if $\xi \in \operatorname{Proj}(G, \alpha)$ such that $\zeta$ is not a constituent of $\xi_{N}$, then $\xi=\varphi^{G}$ for some $\varphi \in \operatorname{Proj}\left(N, \alpha_{N}\right)$.

\section{References}

[1] R. J. Higgs, 'Projective characters of degree one and the inflation-restriction sequence', J. Austral. Math. Soc. Ser. A 46 (1989), 272-280. 
[2] B. Huppert, Endliche Gruppen I (Die Grundlehren der mathematischen Wissenschaften in Einzeldarstellungen 134, Springer-Verlag, Berlin, Heidelberg, New York, 1967).

[3] I. M. Isaacs, Character theory of finite groups (Pure and Applied Mathematics, a series of monographs and textbooks 69, Academic Press, New York, London, 1976).

[4] G. Karpilovsky, Projective representations of finite groups (Monographs and textbooks in pure and applied mathematics 94, Marcel Dekker, New York, Basel, 1985).

[5] G. Karpilovsky, The Schur multiplier (London Mathematical Society Monographs, (N.S.) 2, Oxford University Press, Oxford, New York, 1987).

[6] I. D. MacDonald, 'Commutators and their products', Amer. Math. Monthly 93 (1986), 440-443.

[7] Ruth Mangold, 'Beitrage zur Theorie der Darstellungen endlicher Gruppen durch Kollineationen', Mitt. Math. Sem. Giessen 69 (1966), (ii) 1-44.

\section{University College Dublin}

Belfield

Dublin 4

Ireland 